\title{
Poly(a)morphic portrait of the electrical double layer in ionic liquids
}

\author{
V. Ivaništšev ${ }^{\mathrm{a}, *}$, S. O'Connor ${ }^{\mathrm{a}}$, M.V. Fedorov ${ }^{\mathrm{a}, *}$ \\ ${ }^{a}$ Department of Physics, Scottish Universities Physics Alliance (SUPA), Strathclyde \\ University, \\ John Anderson Building, 107 Rottenrow East, Glasgow, UK G4 ONG.
}

\begin{abstract}
In this paper we present a unified view on charge-driven structural transitions in the electrical double layer in ionic liquids and summarise molecular-scale mechanisms of the ionic liquid structural response to the surface charge.

Keywords: Ionic liquids, Electrical double layer, Molecular simulations
\end{abstract}

\section{Introduction}

Charge/voltage driven structural transitions in the electrical double layer (EDL) in ionic liquids (ILs) have recently attracted large interest in experimental $[1,2,3,4,5,6]$, theoretical $[7]$ and computational $[8,9,10,11,12$, $13,14]$ communities due to the importance of this subject for a variety of IL applications $[15,16]$.

Bazant et al. [7] suggested that general trends in structural transitions in ILs upon surface charging are determined by the crossover between the overscreening and the crowding regime in the EDL structure. In a recent mod-

\footnotetext{
${ }^{*}$ Corresponding author

Email addresses: vladislav.ivanistsev@strath.ac.uk (V. Ivaništšev), maxim.fedorov@strath.ac.uk (M.V. Fedorov)
} 
elling work [11] it was shown that this crossover corresponds to a structural transition from a multilayer (intermediate charges) to an overcrowded structure (high charges; superposition of two or more counter-ion layers) through the formation of a monolayer structure at a certain charge density value. Recently in Ref.[14] it has been suggested that these trends may be universal and are expected to be found in many IL systems (see also [17]). However, this hypothesis is based on theories and models that do not take into account molecular-scale effects of ion geometry and heterogeneous partial charge distribution across the IL molecules, and overall it remains unclear whether the conclusions from the Refs. $[7,11,14]$ are not effects of an oversimplified view on ILs.

Here we make the next step towards rationalising general mechanisms of charge-driven interfacial structural transitions in ILs by investigating and comparing structural behaviour of three different coarse-grained IL models $[18,19,11]$ with the behaviour of a fully atomistic model of 1-butyl-3-

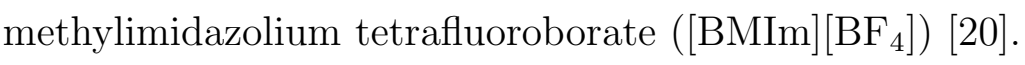

\section{Methods}

\subsection{Simulations}

All simulations were performed using the classical Molecular Dynamics (MD) method in the NVT ensemble at a temperature of $350 \mathrm{~K}$ with the Gromacs 4.5.5 software [21]. The simulation setups (equilibration, length of simulations, system parameters and computational methods) were overall the same to the ones used in our previous works: Ref. [20] (fully atomistic model of $\left.[\mathrm{BMIm}]\left[\mathrm{BF}_{4}\right]\right)$ and Refs. [11] (coarse-grained models). 


\subsubsection{Coarse-grained simulations}

The models represented IL ions as charged Lennard-Jones spheres [18, 11]. Three different models of IL were chosen, with the cation-to-anion diameter $\left(d_{\mathrm{LJ}}\right)$ ratios of $1: 1$ (large anion - LA), $1: 0.8$ (medium anion - MA) and $1: 0.5($ small anion $-\mathrm{SA})$ with constant $d_{\mathrm{LJ}}($ Cation $)=1.0 \mathrm{~nm}$.

The simulated systems represent IL ions confined between two model electrodes $[18,19,11]$. The electrodes consist of 2500 Lennard-Jones spheres with a diameter $\left(d_{\mathrm{LJ}}\right)$ of $0.22 \mathrm{~nm}$ that are arranged on a square lattice with a size of $11 \mathrm{~nm} \times 11 \mathrm{~nm}$, in $x$ and $y$ directions. The distance between the electrodes was chosen to be $54 \mathrm{~nm}, 36 \mathrm{~nm}$ and $24 \mathrm{~nm}$ for the LA, MA and SA systems, respectively. The ion pair number was fixed in all simulations to be equal 1050.

\subsubsection{Fully atomistic simulations}

The system consisted of two rigid graphene slabs with dimensions of 3.408 $\mathrm{nm}$ by $3.4433 \mathrm{~nm}$ separated by a distance of $10.4 \mathrm{~nm} .374[\mathrm{BMIm}]\left[\mathrm{BF}_{4}\right]$ ion pairs were placed between these surfaces and equilibrated. The OPLS-AA force field was used together with partial charges taken from [22] for the IL. The charges were screened by a factor of 0.79 to account for the highfrequency electronic polarisability [20].

\subsection{Analysis}

As in Refs. [11, 14], we define a unified $\kappa$-scale, where the surface charge density $(\sigma)$ is normalised by the maximum charge density that can be stored in a densely packed counter-ion monolayer $\left(\theta_{\mathrm{Ion}}^{\max }\right)$ :

$$
\kappa_{\text {Ion }}=\left|\frac{\sigma}{\theta_{\text {Ion }}^{\max }}\right| \text {. }
$$


Below we use the $\kappa$-scale for generalised analysis with a focus on the regions $0<\kappa_{\text {Anion }}<1$ and $0<\kappa_{\text {Cation }}<2$, where $\kappa_{\text {Cation }}$ corresponds to the negative surface charge density values $(\sigma<0)$ and $\kappa_{\text {Anion }}$ corresponds to the positive values $(\sigma>0)$.

Because the monolayer structure is characterised by smearing of oscillations in the electrostatic potential $\phi(z)$-profiles[11, 14], the value of $\left(\theta_{\mathrm{Ion}}^{\max }\right)$ was extracted from the simulation results at the point of surface charge that corresponds to a linear potential drop. The potential drop at $\kappa_{\text {Ion }}=1$ can be roughly approximated as:

$$
\phi_{\mathrm{ML}}=\frac{d}{\epsilon} \theta_{\mathrm{Ion}}^{\max }
$$

where $\theta_{\mathrm{Ion}}^{\max } \approx e \frac{q_{\mathrm{Ion}}}{r_{\mathrm{Ion}}^{2}}, d$ is the distance between the surface and the monolayer charge planes, $r_{\text {Ion }}$ is ionic radius, $q_{\text {Ion }}$ is ionic charge, $e$ is elementary charge, and $\epsilon$ is permittivity of the monolayer structure. $\theta_{\text {Cation }}^{\max }$ was found to be the same for all three coarse grain-systems $\left(+16 \mu \mathrm{C} / \mathrm{cm}^{2}\right)$ in accordance with the fact that the cation model is the same in all systems. This value equals to the density of one cation per $1 \mathrm{~nm}^{2}$ of the surface that corresponds to the dense coverage of the surface by the cations. $\theta_{\mathrm{Anion}}^{\max }$ values were found to be $-68,-26$ and $-16 \mu \mathrm{C} / \mathrm{cm}^{2}$ for the systems with small, medium and large anions, respectively. These values also correspond to the dense coverage of the surface by the anions. For the atomistic model of $[\mathrm{BMIm}]\left[\mathrm{BF}_{4}\right], \theta_{\mathrm{Anion}}^{\max }$ is $-100 \mu \mathrm{C} / \mathrm{cm}^{2}, \theta_{\text {Cation }}^{\max }$ is $+38 \mu \mathrm{C} / \mathrm{cm}^{2}$.

The restructuring process at different charge densities can be illustrated with the use of the parameter $(\lambda)$ that for an $i$-th ion layer at surface is 
defined as the normalised excess of charge in this layer [14]:

$$
\lambda_{i}=\kappa_{\text {Ion }} \times\left(\left|\frac{\mathrm{cn}_{Q}\left(z_{i}\right)}{\sigma}\right|-1\right),
$$

where $z_{i}$ corresponds to an extremum or to a step height on the ion charge density $-\operatorname{cn}_{Q}(z) / \sigma$-profiles in the $i$-th interval between the two successive interception points $\left|\mathrm{cn}_{Q}(z) / \sigma\right|=0$. In the analysis below we consider only the $\lambda$ parameter of the first ion layer $\left(\lambda_{1}\right)$, therefore the index $i$ is omitted.

The $\kappa_{\text {Ion }}$-scale represents a universal analogue of dimensionless "reaction coordinate" for the EDL restructuring process in response to the surface charge. Analysis of the dependence of $\lambda$ on $\kappa_{\text {Ion }}$ allows to study the evolution of the EDL structure in terms of the charge excess. Namely, an increase of the charge excess in the first interfacial layer $\left(\lambda_{1}\right)$ manifests formation of a multilayer EDL structure, while the decrease of the charge excess indicates the vanishing of the multilayer EDL structure towards the formation of the monolayer structure at $\kappa_{\text {Ion }}=1$.

\section{Results}

Figure 1 presents the dependency of the IL ion number density $\rho_{N}$ from the distance to the electrode $z$ and $\kappa$ in the form of $\rho_{N}(z, \kappa)$ contour maps. These maps illustrate charge-dependent layering of cation (light, red) and anion (dark, blue) for the coarse-grained (left, MA) and the atomistic (right) model IL systems.

Although the compared IL models are quite different from each other, in both cases the contour maps reveal similar features of the IL structural response to the surface charge that are described below. 
As can be seen, the vertical ridges of high ion number density divide the interfacial region into distinct regions of ion accumulation. We refer to the region of counter-ions accumulation closest to the electrode, as the first layer. Counter-ions $\rho_{N}(z, \kappa)$ in the first layer grows upon surface charging while counter-ions become pressed against the surface due to the strong electrostatic attraction. Differently, in the subsequent layer the $\rho_{N}(z, \kappa)$ grows until some saturation at $\kappa_{\text {Ion }} \approx 0.5$ and then decreases until $\kappa_{\text {Ion }}=1.0$. The dotted horizontal lines point to the areas of practical absence of layering around $\kappa_{\text {Ion }}=1.0$ (Figure 1$)$.

To facilitate comparison of different IL systems, in Figure 2, we plot $\lambda$ versus $\kappa$ for cationic and anionic layers. As can be seen, the evolution of the EDL structure upon surface charging is qualitatively the same for all coarsegrained and atomistic models. This implies that the main mechanisms of ion accumulation at the surface are governed mostly by electrostatic interactions and sterical effects. Yet, despite of the apparent general similarity seen in Figures 1 and 2, there is a lamination of the $\rho_{N}\left(z\right.$, Cation) at large $\kappa_{\text {Cation }}$ due to the presence of both parallel and perpendicular orientation of the $[\mathrm{BMIm}]^{+}$ring in the first cationic layer.

\section{Conclusions}

As a summary, Figure 3 illustrates general mechanisms of structural transitions in the EDL which are represented as an ensemble of charge-dependent poly(a)morphic structures. These mechanisms can be described by the formation and mutual transformation of ionic bilayer (D,E), multilayer $(\mathrm{C}, \mathrm{F})$ and monolayer $(\mathrm{B}, \mathrm{G})$ structures followed by crowding of the counter-ions at 
high surface charges $(\mathrm{A}, \mathrm{H})$.

D,E: Ionic bilayers of cations and anions that are formed at small surface charges $\kappa \approx 0$. Analysis of the MD simulations results reveals that at small absolute $\sigma$ values the first layer consists of two correlated subsystems - anionic and cationic - that form the ionic bilayer. Upon surface charging, the cationic and anionic subsystems become separated in space due to depletion of the co-ions and enrichment of the counter-ions near the surface. The anionic and cationic subsystems become completely separated by $\kappa \approx 0.5$ when the multilayer structure is formed.

C,F: Multilayered structures formed at intermediate $\kappa$-values that are composed from well-distinguishable layers of cations and anions that alternate in the normal direction (see Figure 1). These multilayered structures are characterised by maximal values of the charge excess parameter $(\lambda)$ (see Figure 2).

B,G: Cationic (B) and anionic $(\mathrm{G})$ monolayer structures formed at $\kappa$-values close to 1.0. In the cationic monolayer most of the $[\mathrm{BMIm}]^{+}$rings lie parallel to the surface and few of the $[\mathrm{BMIm}]^{+}$rings orient perpendicular to the surface. Noteworthy that the reorientation of a small part of the $[\mathrm{BMIm}]^{+}$rings happens for the same reason as the displacement of the tails from the surface - in order to provide higher charge density in the ionic layer closer to the surface. The tails are pulled away from the surface at $\kappa>0.5$ forming a flexible sublayer. This observation is in agreement with experimental evidences of formation of a similar 
intermediate layer [23] in a confined pyrrolidinium-based IL.

$\mathbf{A}, \mathbf{H}$ : Crowded structures formed by cations (A) and anions (H) at high values of $\kappa_{\text {Ion }}\left(\kappa_{\text {Ion }}>1.0\right)$ when the net counter charge cannot be provided by a single dense layer of counter-ions [7]. We point to the molecular details of this phenomenon for ions with complex molecular geometry like the $[\mathrm{BMIm}]^{+}$cations considered in this study: in the molecular simulations a higher counter-charge can be accumulated either by the formation of a distinguishable second sublayer of [BMIm] $]^{+}$ cations (leading to crowding) or by reorientation of the $[\mathrm{BMIm}]^{+}$rings from parallel to perpendicular orientation relative to the surface (due to electrostriction).

The visualised reorientation of the alkyl chains and the $[\mathrm{BMIm}]^{+}$ring (Figure 3B-D) is in agreement with recent spectroscopic studies [24]. Moreover, the molecular representation of the bilayer-to-multilayer restructuring (Figure 3C-F) supports atomic force microscopy (AFM) insights into the EDL structure in ILs $[5,25,2,26]$. First, using eq. 2 with $\epsilon=1.6$ we estimate the potential of the monolayer formation of common $[\mathrm{BPyr}]^{+},[\mathrm{TFSI}]^{-}$, $[\mathrm{FAP}]^{-}$to be $-3.1 \mathrm{~V},+2.9 \mathrm{~V}$ and $+2.7 \mathrm{~V}$, respectively. Consequently, we conclude that the electrode potential range used in experiments $[5,25,2,26]$ corresponds to the $\kappa_{\text {Ion }}$ values between 0 and $0.5-0.7$. In both MD simulations and in AFM experiments $[5,26]$, upon $\kappa_{\text {Cation }} \rightarrow 0.5$, the number of interfacial layers is increasing (Figure 1); at negative surface charge densities the thickness of the first layer varies due to the reorientation of the cation ring (Figure 3C); and at positive surface charge densities the thickness of 
the first layer remains constant, although, the alkyl tails may penetrate the anionic sublayer (Figure 3E).

The presented results also prove that the earlier ideas on over-screening to overcrowding structural transitions [7] obtained on simple IL models $[11,14]$ are still generally valid even when the molecular structure of IL ions is taken into account.

\section{Acknowledgement}

We acknowledge the supercomputing support from ARCHIE-WeSt High Performance Computer centre (www.archie-west.ac.uk, EPSRC grant no. $\mathrm{EP} / \mathrm{K} 000586 / 1)$. 


\section{References}

[1] Y. Liu, Y. Zhang, G. Wu, J. Hu, Coexistence of liquid and solid phases of bmim-PF6 ionic liquid on mica surfaces at room temperature, J. Am. Chem. Soc. 128 (23) (2006) 7456-7457.

[2] J. Sweeney, F. Hausen, R. Hayes, G. B. Webber, F. Endres, M. W. Rutland, R. Bennewitz, R. Atkin, Control of nanoscale friction on gold in an ionic liquid by a potential-dependent ionic lubricant layer, Phys. Rev. Lett. 109 (15) (2012) 155502-5.

[3] H. Zhou, M. Rouha, G. Feng, S. S. Lee, H. Docherty, P. Fenter, P. T. Cummings, P. F. Fulvio, S. Dai, J. McDonough, V. Presser, Y. Gogotsi, Nanoscale perturbations of room temperature ionic liquid structure at charged and uncharged interfaces, ACS Nano 6 (11) (2012) 9818-9827.

[4] F. Buchner, K. Forster-Tonigold, B. Uhl, D. Alwast, N. Wagner, A. Groß, R. J. Behm, Towards the microscopic identification of anions and cations at the ionic liquid | ag(111) interface: A combined experimental and theoretical investigation, ACS Nano 7 (2013) 7773-7784.

[5] H. Li, R. J. Wood, F. Endres, R. Atkin, Influence of alkyl chain length and anion species on ionic liquid structure at the graphite interface as a function of applied potential, J. Phys.: Condens. Matter 26 (28) (2014) 284115.

[6] T. Carstens, R. Gustus, O. Höfft, N. Borisenko, F. Endres, H. Li, R. J. Wood, A. J. Page, R. Atkin, Combined STM, AFM, and DFT study of the highly ordered pyrolytic graphite/1-octyl-3-methyl-imidazolium 
bis(trifluoromethylsulfonyl)imide interface, J. Phys. Chem. C 118 (20) (2014) 10833-10843.

[7] M. Z. Bazant, B. D. Storey, A. A. Kornyshev, Double layer in ionic liquids: Overscreening versus crowding, Phys. Rev. Lett. 106 (4) (2011) 046102-4.

[8] S. Tazi, M. Salanne, C. Simon, P. Turq, M. Pounds, P. A. Madden, Potential-induced ordering transition of the adsorbed layer at the ionic Liquid/Electrified metal interface, J. Phys. Chem. B 114 (25) (2010) 8453-8459.

[9] E. Paek, A. J. Pak, G. S. Hwang, A computational study of the interfacial structure and capacitance of graphene in [BMIM] $[\mathrm{PF} 6]$ ionic liquid, J. Electrochem. Soc. 160 (1) (2013) A1-A10.

[10] S. A. Kislenko, R. H. Amirov, I. S. Samoylov, Molecular dynamics simulation of the electrical double layer in ionic liquids, J. Phys.: Conf. Ser. 418 (1) (2013) 012021-8.

[11] K. Kirchner, T. Kirchner, V. Ivaništšev, M. Fedorov, Electrical double layer in ionic liquids: Structural transitions from multilayer to monolayer structure at the interface, Electrochim. Acta 110 (2013) 762-771.

[12] C. Merlet, B. Rotenberg, P. A. Madden, M. Salanne, Computer simulations of ionic liquids at electrochemical interfaces, Phys. Chem. Chem. Phys. 15 (38) (2013) 15781-15792.

[13] C. Merlet, D. T. Limmer, M. Salanne, R. van Roij, P. A. Madden, 
D. Chandler, B. Rotenberg, The electric double layer has a life of its own, J. Phys. Chem. C.

[14] V. Ivaništšev, M. V. Fedorov, Interfaces between charged surfaces and ionic liquids: Insights from molecular simulations, Interface 23 (1) (2014) 65-69.

[15] F. Endres, O. Höfft, N. Borisenko, L. H. Gasparotto, A. Prowald, R. AlSalman, T. Carstens, R. Atkin, A. Bund, S. Z. El Abedin, Do solvation layers of ionic liquids influence electrochemical reactions?, Phys. Chem. Chem. Phys. 12 (8) (2010) 1724-1732.

[16] M. V. Fedorov, A. A. Kornyshev, Ionic liquids at electrified interfaces, Chem. Rev. 114 (5) (2014) 2978-3036.

[17] A. A. Kornyshev, R. Qiao, Three-dimensional double layers, J. Phys. Chem. C.

[18] M. V. Fedorov, A. A. Kornyshev, Ionic liquid near a charged wall: Structure and capacitance of electrical double layer, J. Phys. Chem. B 112 (38) (2008) 11868-11872.

[19] M. V. Fedorov, A. A. Kornyshev, Towards understanding the structure and capacitance of electrical double layer in ionic liquids, Electrochim. Acta 53 (23) (2008) 6835-6740.

[20] V. Ivaništšev, M. V. Fedorov, R. M. Lynden-Bell, Screening of Ion-Graphene electrode interactions by ionic liquids: The effects of liquid structure, J. Phys. Chem. C 118 (11) (2014) 5841-5847. 
[21] B. Hess, C. Kutzner, D. van der Spoel, E. Lindahl, GROMACS 4: Algorithms for highly efficient, load-balanced, and scalable molecular simulation, J. Chem. Theory Comput. 4 (3) (2008) 435-447.

[22] S. V. Sambasivarao, O. Acevedo, Development of OPLS-AA force field parameters for 68 unique ionic liquids, J. Chem. Theory Comput. 5 (4) (2009) 1038-1050.

[23] A. M. Smith, K. R. J. Lovelock, N. N. Gosvami, P. Licence, A. Dolan, T. Welton, S. Perkin, Monolayer to bilayer structural transition in confined pyrrolidinium-based ionic liquids, J. Phys. Chem. Lett. 4 (2013) $378-382$.

[24] S. Baldelli, Interfacial structure of room-temperature ionic liquids at the Solid-Liquid interface as probed by sum frequency generation spectroscopy, J. Phys. Chem. Lett. 4 (2) (2013) 244-252.

[25] H. Li, F. Endres, R. Atkin, Effect of alkyl chain length and anion species on the interfacial nanostructure of ionic liquids at the au(111)-ionic liquid interface as a function of potential, Phys. Chem. Chem. Phys. 15 (35) (2013) 14624-14633.

[26] R. Hayes, N. Borisenko, M. K. Tam, P. C. Howlett, F. Endres, R. Atkin, Double layer structure of ionic liquids at the au(111) electrode interface: An atomic force microscopy investigation, J. Phys. Chem. C 115 (14) (2011) 6855-6863. 

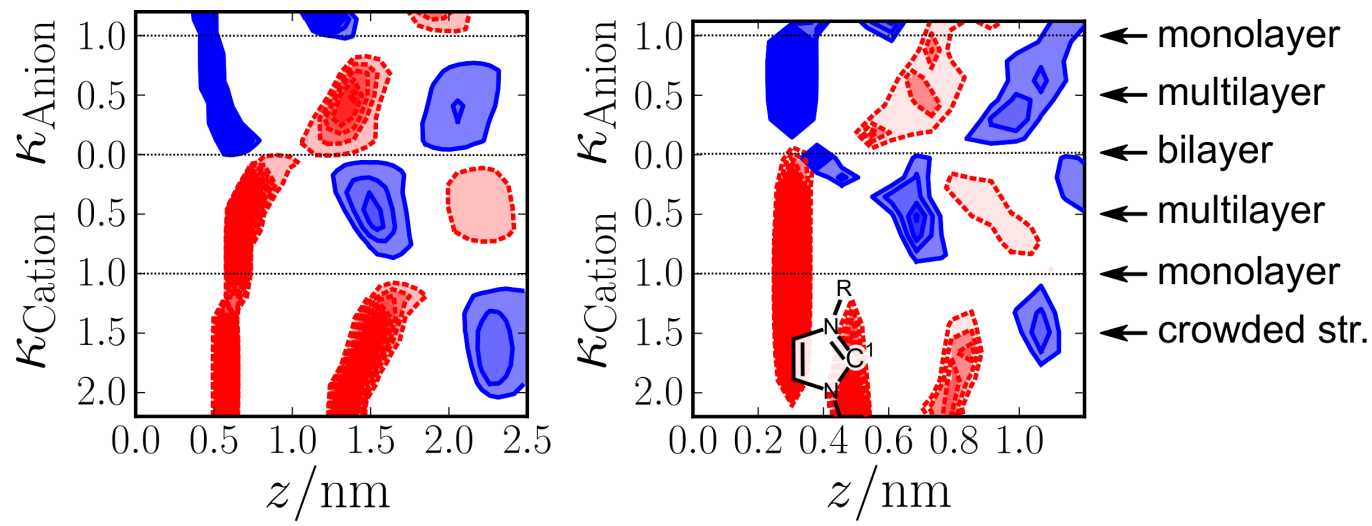

Figure 1: The figure presents the ion number density $\rho_{N}(z, \kappa)$ contour maps that illustrate charge-dependent layering of cation (light, red) and anion (dark, blue) for the MA (left) and the $[\mathrm{BMIm}]\left[\mathrm{BF}_{4}\right]$ (right) models. The contour interval equals to $\rho_{\text {bulk }}$, the first contour starts at $1.5 \rho_{\text {bulk }}(\mathrm{MA})$ and $2.5 \rho_{\text {bulk }}\left([\mathrm{BMIm}]\left[\mathrm{BF}_{4}\right]\right)$, and the $\rho_{N}(z, \kappa)$ peaks are cut at

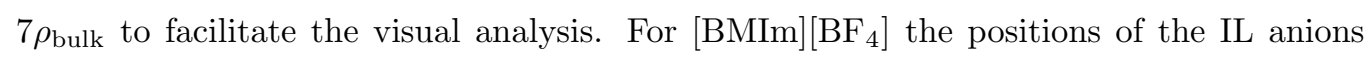
and cations are assigned to the centres of $\mathrm{C}^{1}$ and $\mathrm{B}$ atoms respectively; the picture of [BMIm] $^{+}$indicates that the lamination of the $\rho_{N}\left(z\right.$, Cation) at large $\kappa_{\text {Cation }}$ values is due to the presence of parallel and perpendicular (shown) orientation of the aromatic ring in the first cationic layer at high surface charges. 


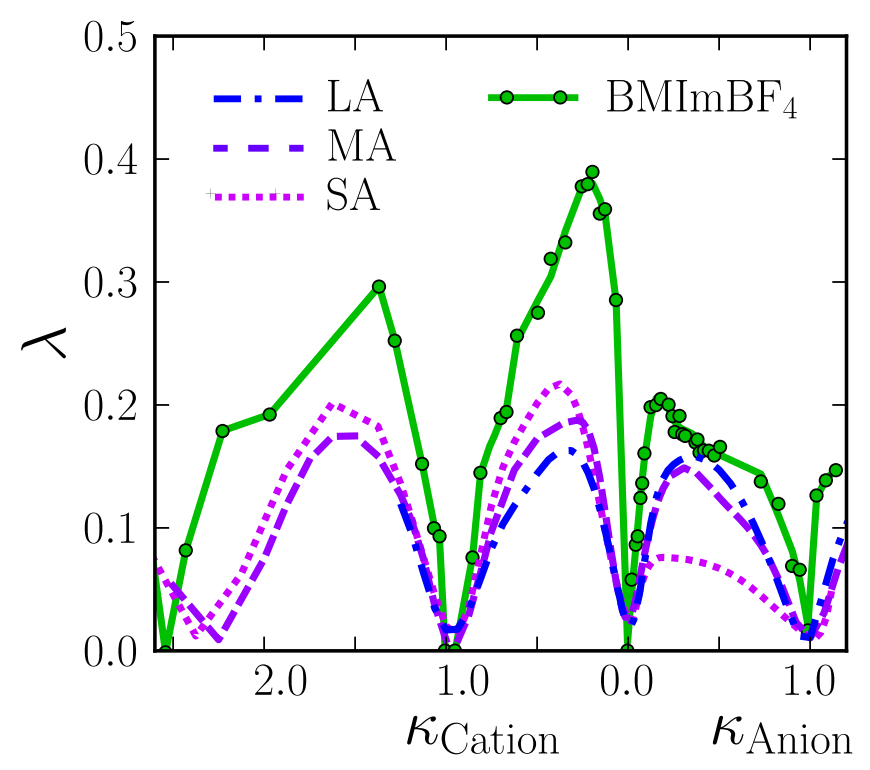

Figure 2: Variation of the normalised charge excess in the first interfacial layer $(\lambda)$ with $\kappa$ for the four model ILs studied in this work. The figure illustrates the overall similarity of the charge-induced EDL restructuring in these different model ILs. The $\kappa_{\text {Ion }}$ values of 1.0 correspond to the $\theta_{\mathrm{Ion}}^{\max }$ values of the charge density $\sigma . \lambda$ minimum at $\kappa \approx 2.3$ indicates the formation of a crowded layer of cations which accommodates more counter-ions that is expected from a superposition of two dense monolayers; that happens due to the squeezing and reorientation of the cations in the strong electric field at these high charges. 


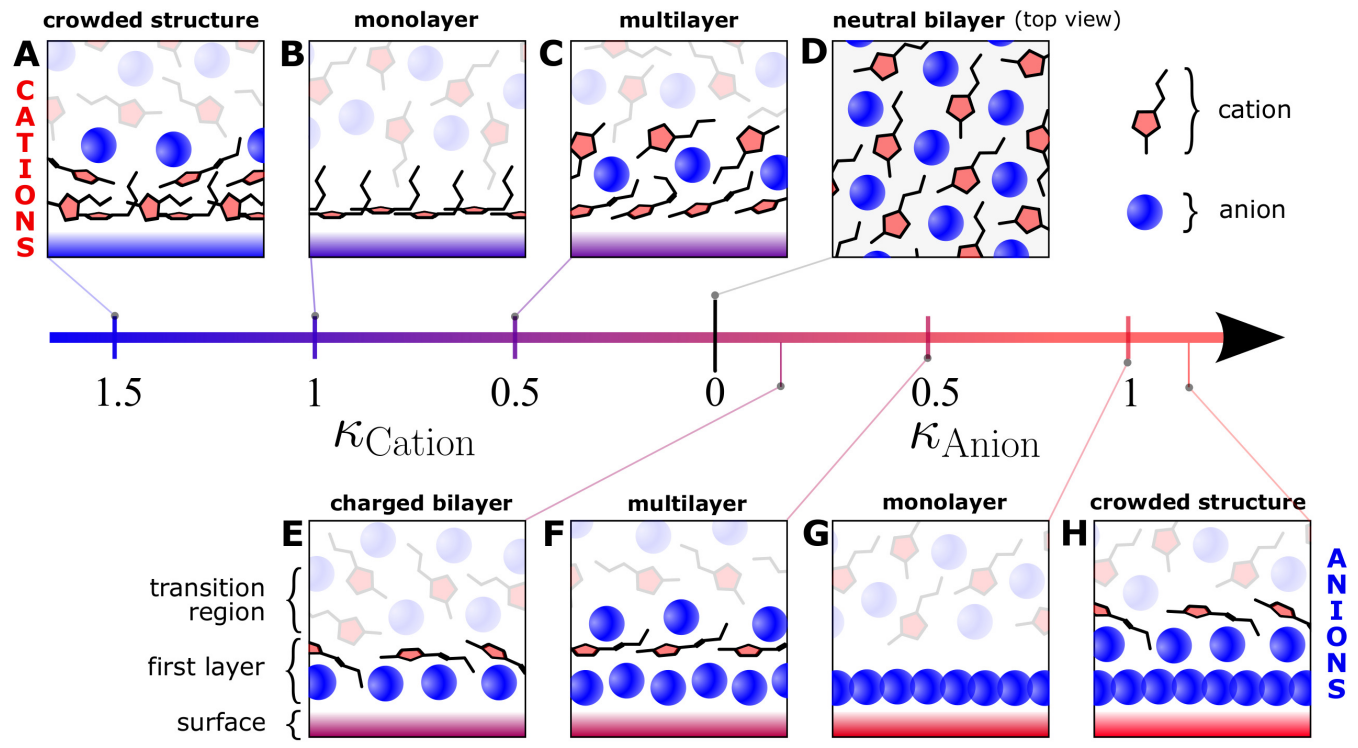

Figure 3: Schematic representation of cations (red pentagons with black tails) and anions (blue balls) packing and orientations close to the electrode (coloured rectangle) at different $\kappa$-values. Links between the shown structures and the position on the $\kappa$-scale are indicated with lines. In terms of cation and anion packing, the EDL structures formed upon surface charging can be roughly classified as ionic bilayer $(\mathrm{D}, \mathrm{E})$, multilayer $(\mathrm{C}, \mathrm{F})$, monolayer $(\mathrm{B}, \mathrm{G})$ and crowded polyamorphic structures $(\mathrm{A}, \mathrm{H})$. 